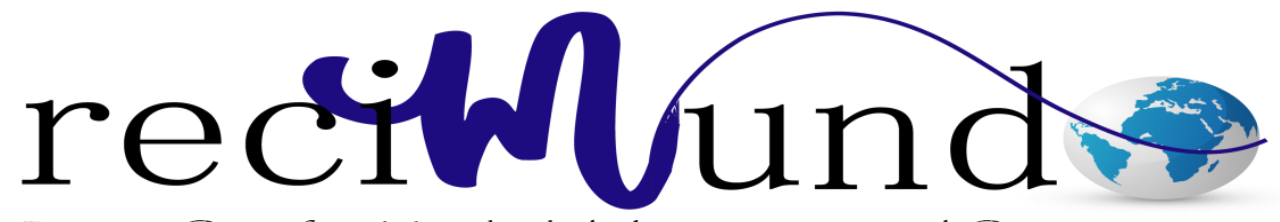

Revista Científica Mundo de la Investigación y el Conocimiento

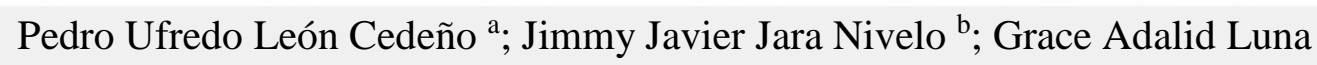
Yerovi $^{\mathrm{c}}$

Propuesta de flujo efectivo con financiamiento utilizando bono o acciones para la empresa de fertilizante

Revista Científica Mundo de la Investigación y el Conocimiento. Vol. 2 núm., especial, mayo, ISSN: 2588-073X, 2018, pp. 924-941

DOI: 10.26820/recimundo/2.esp.2018.924-941

Editorial Saberes del Conocimiento

Recibido: 20/12/2017

Aceptado: 26/02/2018
a. Universidad de Guayaquil.
b. Universidad de Guayaquil.
c. Universidad de Guayaquil. 


\section{Propuesta de flujo efectivo con financiamiento utilizando bono o acciones para la empresa de fertilizante \\ Vol. 2, núm. Esp., (2018) \\ Pedro Ufredo León Cedeño; Jimmy Javier Jara Nivelo; Grace Adalid Luna Yerovi}

\section{RESUMEN}

Introducción: A pesar del avance pujante de los últimos años, y al igual que la mayoría de los países latinoamericanos, su capital sigue dependiendo de las exportaciones de materias primas y el petróleo es la principal fuente de riqueza del país. Ecuador, como todos los países de América Latina, se independizó porque quería tener poder político y libertad para desarrollar sus actividades económicas; los criollos consideraban injusto estar excluidos de la política y de las decisiones financieras. Objetivo: Elaborar Flujos Efectivos para lograr financiamiento a largo plazo de una empresa de fertilizantes. Propuesta: La importancia de un flujo de caja se afirma acertadamente en la expresión común de liquidez. La premisa de esto es que el tener dinero en efectivo que pone en una posición más estable a la empresa generando una mejor liquidez. Si bien se puede pedir dinero prestado a veces, el dinero en efectivo que se genera, ofrece una mayor protección contra los impagos de préstamos o hipotecas. El flujo de caja es distinta de la posición de efectivo. Tener dinero en efectivo a la mano es crítica, pero el flujo de efectivo indica una capacidad permanente para generar y utilizar el dinero en efectivo. Conclusiones: Se pudo constatar los problemas financieros de la empresa, el implementar una herramienta que ayude en la gestión de efectivo, de esta manera la empresa obtenga una mejor liquidez al finalizar el periodo contable.

Palabras claves: Flujo efectivo; empresa de fertilizantes; periodo contable. 


\title{
Propuesta de flujo efectivo con financiamiento utilizando bono o acciones para la empresa de fertilizante
}

Vol. 2, núm. Esp., (2018)

Pedro Ufredo León Cedeño; Jimmy Javier Jara Nivelo; Grace Adalid Luna Yerovi

\begin{abstract}
Introduction: Despite the booming progress of recent years, and like most Latin American countries, its capital continues to depend on exports of raw materials and oil is the main source of wealth for the country. Ecuador, like all the countries of Latin America, became independent because it wanted to have political power and freedom to develop its economic activities; the criollos considered it unjust to be excluded from politics and financial decisions. Objective: Develop Cash Flows to obtain long-term financing from a fertilizer company. Proposal: The importance of a cash flow is correctly stated in the common expression of liquidity. The premise of this is that having cash that puts the company in a more stable position generates better liquidity. While you can borrow money at times, the cash that is generated, offers greater protection against defaults on loans or mortgages. The cash flow is different from the cash position. Having cash on hand is critical, but the cash flow indicates a permanent ability to generate and use the cash. Conclusions: It was possible to verify the financial problems of the company, to implement a tool that helps in cash management, in this way the company obtains a better liquidity at the end of the accounting period.
\end{abstract}

Keywords: Cash flow; fertilizer company; accounting period. 


\section{Propuesta de flujo efectivo con financiamiento utilizando bono o acciones para la empresa de fertilizante \\ Vol. 2, núm. Esp., (2018) \\ Pedro Ufredo León Cedeño; Jimmy Javier Jara Nivelo; Grace Adalid Luna Yerovi}

\section{Introducción.}

El propósito del estado de flujos de efectivo o estado de flujos de efectivo es proporcionar información sobre los ingresos brutos de la empresa y los pagos por un período de tiempo especificado. (Durán, 2011) (Finnerty, 1998)

Los ingresos brutos y los pagos brutos serán reportados en el estado de flujos de efectivo de acuerdo con una de las siguientes clasificaciones: las actividades de operación, actividades de inversión y actividades de financiación. El cambio neto de estas tres clasificaciones debe ser igual al cambio en efectivo y equivalentes de efectivo de una empresa durante el período del informe. (Garrido \& Granados, 2004)

Se escogió el financiamiento de la venta de acciones por el motivo de crear pequeñas sociedades y relaciones comerciales ya sea con otras empresas, personas naturales o jurídicas, debido a esto la imagen de la empresa crecerá o penetrará en nuevos mercados. La propuesta tiene como objetivo ser una herramienta que ayude a la organización a gestionar los ingresos y salidas de capital que se efectúen con la actividad principal de la empresa. (Vargas Soto, 2007) (Páez, 2007)

\section{Desarrollo de la Propuesta.}

Las características de flujo de caja es que deben contar los niveles de ventas y los gastos, que se resumen de la siguiente manera: 


\section{Propuesta de flujo efectivo con financiamiento utilizando bono o acciones para la empresa de fertilizante}

Vol. 2, núm. Esp., (2018)

Pedro Ufredo León Cedeño; Jimmy Javier Jara Nivelo; Grace Adalid Luna Yerovi

- Las ventas en efectivo generan flujo de efectivo inmediato. Tener en cuenta que las devoluciones de ventas y ajustes de precio se realizan después del ingreso de valor de venta en el flujo de caja.

- Las ventas a crédito no generan flujo de efectivo inmediato. No se registra ningún valor hasta que no se haya realiza las respectivas cobranzas de cuentas por cobrar y se conviertan en dinero en efectivo que ingrese en la empresa.

- El gasto de depreciación se registra mediante la reducción del valor en libros de un activo y no implica gasto de efectivo en el período en que se registra. La empresa paga en efectivo cuando se adquirió el activo. (El gasto de amortización de los activos intangibles es lo mismo).

Las características favorables de flujo de efectivo es que los ingresos por suscripción y estructura de costos, combinadas con las características positivas de capital de trabajo y los bajos requerimientos de inversión en capital, permitirán que históricamente se genere un flujo de efectivo importante.

En la evaluación de los resultados, se centrarán en varios datos financieros y operativos esenciales, incluyendo el valor anualizado del contrato, las tasas de renovación, las ventas a nuevos clientes, los ingresos no recurrentes, etc.

Importancia del flujo de efectivo

El flujo de caja es el cambio neto en la posición de efectivo de la empresa desde un período al siguiente. Si se genera ingresos generando menos costos se obtendrá un flujo de caja 


\section{Propuesta de flujo efectivo con financiamiento utilizando bono o acciones para la empresa de fertilizante}

Vol. 2, núm. Esp., (2018)

Pedro Ufredo León Cedeño; Jimmy Javier Jara Nivelo; Grace Adalid Luna Yerovi

positivo. Si se tiene un flujo de caja negativo quiere decir que la empresa tiene más gastos que ingresos y no está generando utilidad.

La importancia de un flujo de caja se afirma acertadamente en la expresión común de liquidez. La premisa de esto es que el tener dinero en efectivo que pone en una posición más estable a la empresa generando una mejor liquidez. Si bien se puede pedir dinero prestado a veces, el dinero en efectivo que se genera, ofrece una mayor protección contra los impagos de préstamos o hipotecas. El flujo de caja es distinta de la posición de efectivo. Tener dinero en efectivo a la mano es crítica, pero el flujo de efectivo indica una capacidad permanente para generar y utilizar el dinero en efectivo.

\section{Crecimiento del negocio}

Con el flujo de caja positivo, la comodidad y la capacidad de la empresa para invertir en el crecimiento. La construcción de nuevas ubicaciones, la inversión en investigación y desarrollo, la renovación de la infraestructura, la mejora de la tecnología, la mejora de la formación y de la compra de más activos e inventario son algunas de las maneras en que el negocio puede crecer y mejorar con un fuerte flujo de caja.

\section{El flujo de caja para la toma de decisiones}

Los gestores, inversores, acreedores y todo personal administrativo o societario necesitan información sobre el efectivo y los flujos de modo que puedan tomar decisiones. La importante fuente de información financiera sobre la organización es el estado de flujos de efectivo. 


\section{Propuesta de flujo efectivo con financiamiento utilizando bono o acciones para la empresa de fertilizante}

Vol. 2, núm. Esp., (2018)

Pedro Ufredo León Cedeño; Jimmy Javier Jara Nivelo; Grace Adalid Luna Yerovi

El estado de flujo de efectivo, es uno de los cuatro estados financieros básicos, que proporcionará información sobre las cantidades y tipos de efectivo de la entidad durante el período de flujos. El propósito de la propuesta es diseñar y examinar el tipo de información de manejo de capital que proporciona para la empresa.

1. Describir el tipo de información que se incluye en una caja el estado de flujos, cómo se organiza y cómo es útil para la toma de decisiones.

2. Describir los diferentes tipos de flujos de efectivo que sean importantes para los tomadores de decisiones y la forma de dinero en efectivo

3. Explicar los flujos de efectivo procedentes de los tipos comunes de transacciones y describen la forma en que se presentan en la el estado de flujos de efectivo.

4. Explicar cómo los responsables de las decisiones analizan las fuentes de efectivo y los usos enumerados en el estado de flujos de efectivo, y describir que relaciones comerciales a menudo se utilizan en el análisis de flujos de efectivo.

El flujo de caja está relacionado con actividades operacionales como:

- Las entradas de efectivo y las ventas de bienes y servicios

- Cobros en concepto de ganancias en inversiones en valores (intereses y dividendos)

- Los pagos a los proveedores

- $\quad$ Los pagos a los empleados

- Los pagos de intereses 


\section{Propuesta de flujo efectivo con financiamiento utilizando bono o acciones para la empresa de fertilizante}

Vol. 2, núm. Esp., (2018)

Pedro Ufredo León Cedeño; Jimmy Javier Jara Nivelo; Grace Adalid Luna Yerovi Los pagos de impuestos

Los flujos de efectivo relacionados con actividades de inversión son:

- Las entradas de efectivo de la venta de valores de otras empresas

- Cobros por venta de activos productivos

- Los pagos por la compra de valores de otras empresas

- Los pagos en el momento de la compra para la adquisición de activos productivos

Flujos de efectivo relacionados con actividades de financiación:

- Las ganancias de capital de la emisión de acciones u otros valores de renta variable

- Las ganancias de la emisión de títulos de deuda o la obtención de préstamos (distintos de los créditos comerciales)

- Los pagos de readquisición de capital social u otros valores de renta variable de la entidad

- Los pagos por la retirada de los títulos de deuda (excluyendo intereses)

- Los pagos del principal de los préstamos (distintos de los acreedores comerciales)

- $\quad$ Los pagos de dividendos

Evaluación de flujo de efectivo de operaciones

La empresa debe reportar la información detallada sobre el flujo de caja, debido a que el flujo total de efectivo de operación es importante, que proporciona los datos que permite a los 


\section{Propuesta de flujo efectivo con financiamiento utilizando bono o acciones para la empresa de fertilizante}

Vol. 2, núm. Esp., (2018)

Pedro Ufredo León Cedeño; Jimmy Javier Jara Nivelo; Grace Adalid Luna Yerovi

tomadores de decisiones desarrollar una mejor comprensión de la situación de caja de la empresa y, a su vez, hacer mejores proyecciones de los flujos de efectivo futuros.

A partir de la sección de operaciones del estado de flujos de efectivo con los ingresos netos proporciona una comparación entre los resultados y flujos de efectivo de base devengada y el estado de flujos de efectivo para la cuenta de resultados.

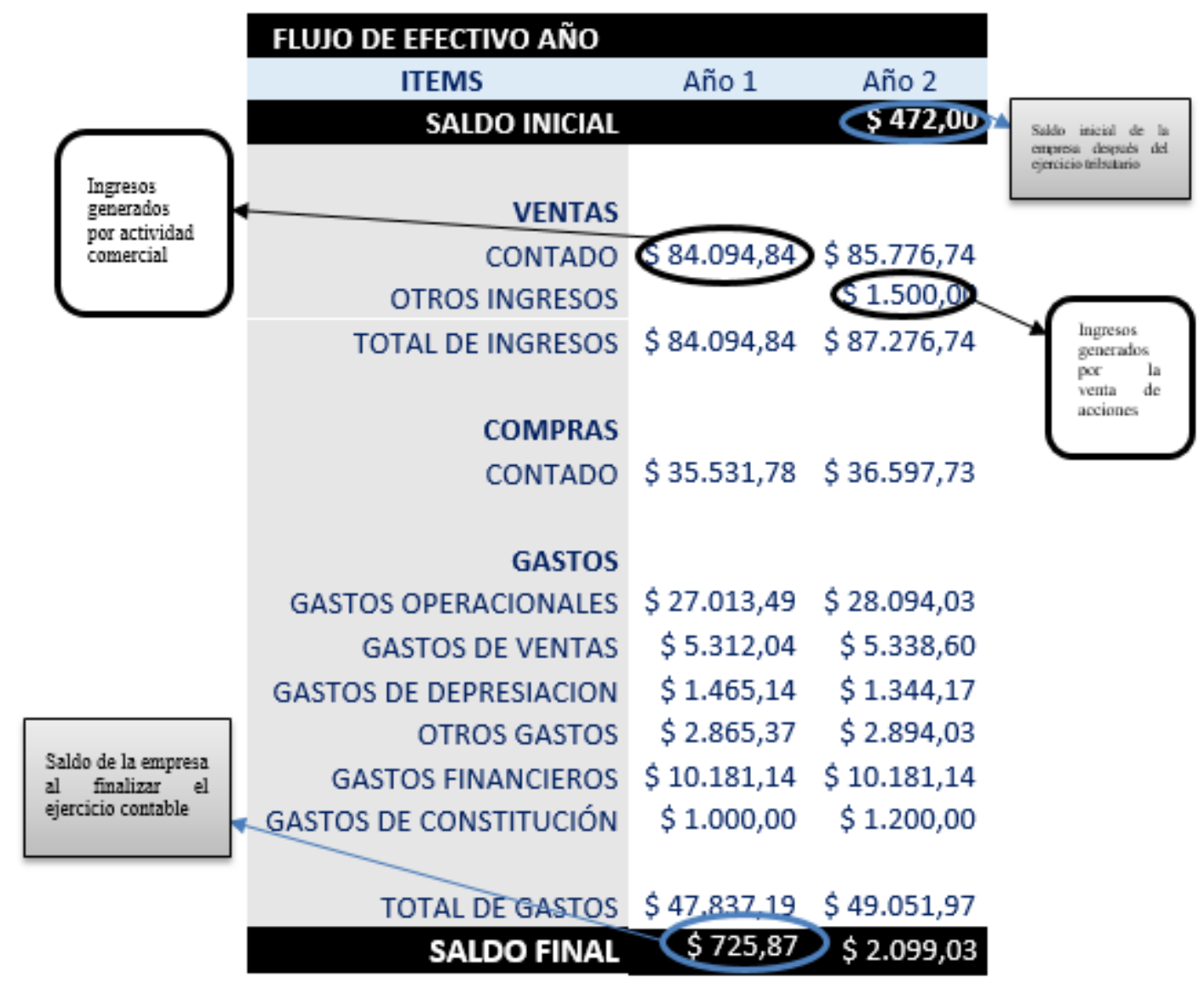

Tabla $N^{\circ} 1$ Modelo de flujo de efectivo 


\section{Propuesta de flujo efectivo con financiamiento utilizando bono o acciones para la empresa de fertilizante}

Vol. 2, núm. Esp., (2018)

Pedro Ufredo León Cedeño; Jimmy Javier Jara Nivelo; Grace Adalid Luna Yerovi

La tabla anterior detalla el diseño de un flujo de caja el cual es sencillo y apropiado para la adecuada toma de decisiones por parte del personal competente, en la tabla se puede observar el total de ingresos por actividad de la empresa, otros ingresos que puedan obtener en este caso por la venta de acciones, y los dos diferentes gastos que realizara para obtener los niveles de venta que reflejan los estados financieros de la empresa.

\section{Ejercicio de flujo de cajas}

Objetivos del ejercicio

- Definir el funcionamiento básico del flujo de caja

- $\quad$ Estructurar los niveles básicos del flujo de caja como son la operación, inversión y financiamiento.

- $\quad$ Elaborar el flujo de caja a partir de los estados financieros de la empresa.

- $\quad$ Elaborar proyecciones del flujo de caja a 10 años.

- Diagnosticar el método de inversión que beneficiará a la empresa.

- $\quad$ Emitir el informe sobre la operación, inversión y financiamiento.

Análisis del flujo de caja

El estado de flujo de caja organiza los datos brindados por los estados financieros de la empresa, en él se detallan los ingresos y salidas de efectivo que se originaron por la actividad principal de la empresa durante un periodo contable. La información es de gran importancia para la parte administrativa y societaria, debido a que brinda la información para la adecuada toma de decisiones y la situación financiera de la empresa. 


\section{Propuesta de flujo efectivo con financiamiento utilizando bono o acciones}

para la empresa de fertilizante

Vol. 2, núm. Esp., (2018)

Pedro Ufredo León Cedeño; Jimmy Javier Jara Nivelo; Grace Adalid Luna Yerovi

Mediante la información obtenida de los estados financieros se pueden proyectar flujos de efectivos positivos a partir de proyecciones con relación a la actividad comercial. Otro diagnostico puede realizarse mediante la capacidad de captar fuentes de financiamiento, siempre y cuando esta obtenga una liquidez y flujo positivos.

Tabla $N^{\circ} 2$ Entradas y salidas de efectivo por actividades

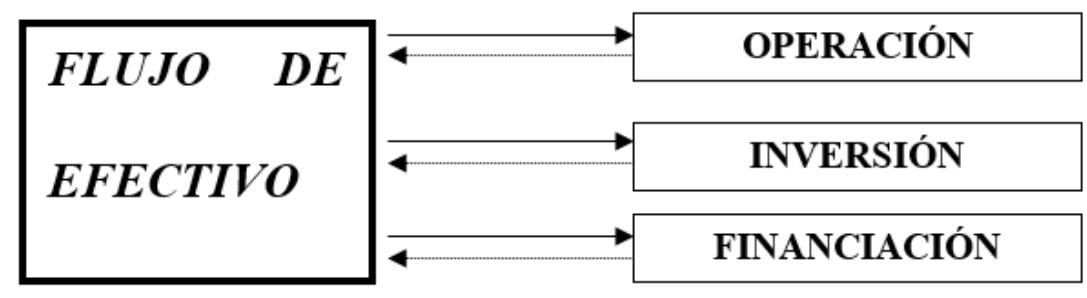

Modelo básico para el estado de Flujo de Efectivo

El estado de flujo de caja presenta el saldo final que obtiene la empresa al final del periodo. Se diseña atendiendo las entradas y salidas de efectivo de manera general o divido en actividades operativas por medios directos e indirectos. El diseño de esta estado puede ser escalando, la fórmula para determinar el saldo final es la siguiente:

ENTRADAS - SALIDAS + SALDO INICIAL $=$ SALDO FINAL 


\section{Propuesta de flujo efectivo con financiamiento utilizando bono o acciones para la empresa de fertilizante}

Vol. 2, núm. Esp., (2018)

Pedro Ufredo León Cedeño; Jimmy Javier Jara Nivelo; Grace Adalid Luna Yerovi

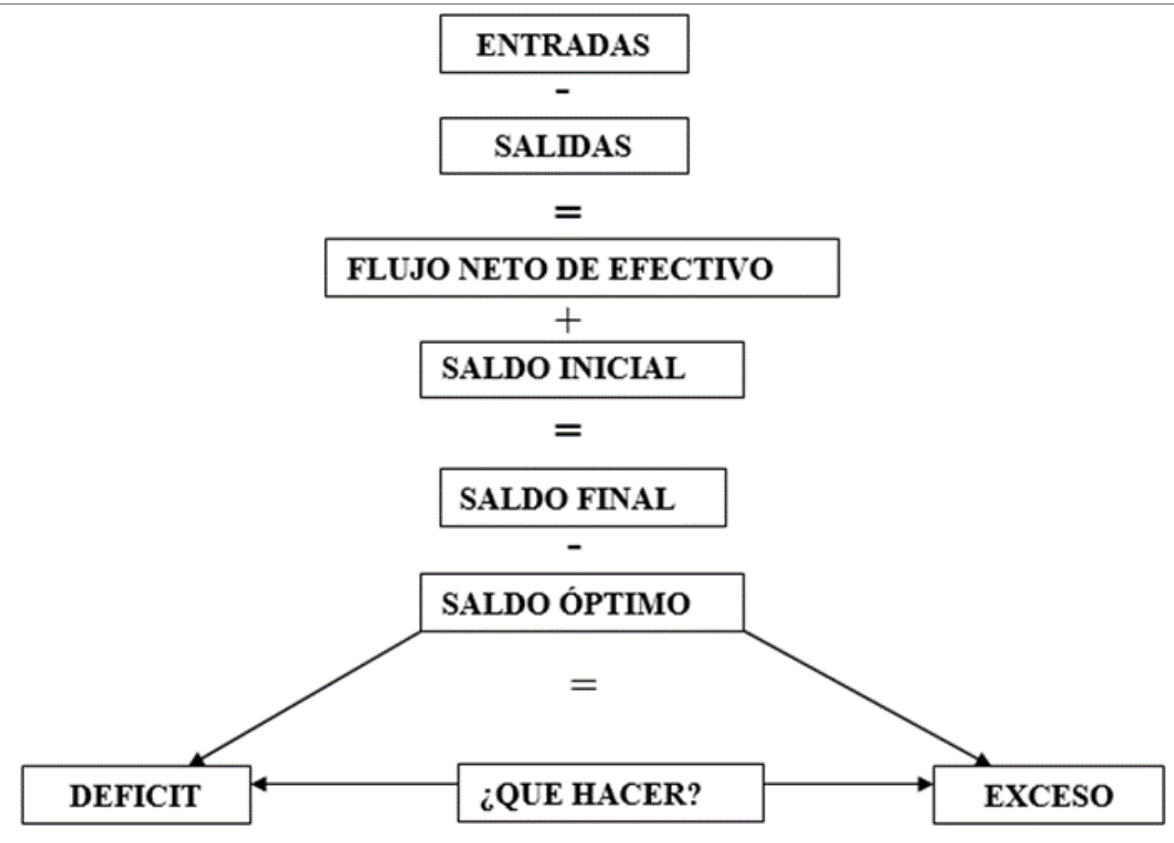

Gráfico $N^{\circ} 2$ Flujograma

Para definir el saldo óptimo de efectivo se debe determinar el ciclo operativo, para lo cual se impone el estudio de los estados financieros, definir las políticas de inventario y créditos que se otorgan a los clientes. Si la empresa no posee la capacidad de generar un saldo optimo, presentará un déficit de caja o capital. Si al termino del ejercicio la empresa presenta saldo optimo se tomará como recursos financiero la venta de acciones, caso contrario la parte administrativa debe realizar la respectiva toma de decisiones para mejorar los índices de ingresos y/o reducir los índices de costos y gastos. 


\section{Propuesta de flujo efectivo con financiamiento utilizando bono o acciones para la empresa de fertilizante}

Vol. 2, núm. Esp., (2018)

Pedro Ufredo León Cedeño; Jimmy Javier Jara Nivelo; Grace Adalid Luna Yerovi

\section{Gráfico $N^{\circ} 3$ ¿Qué hacer cuando se obtiene déficit o superávit de efectivo?}
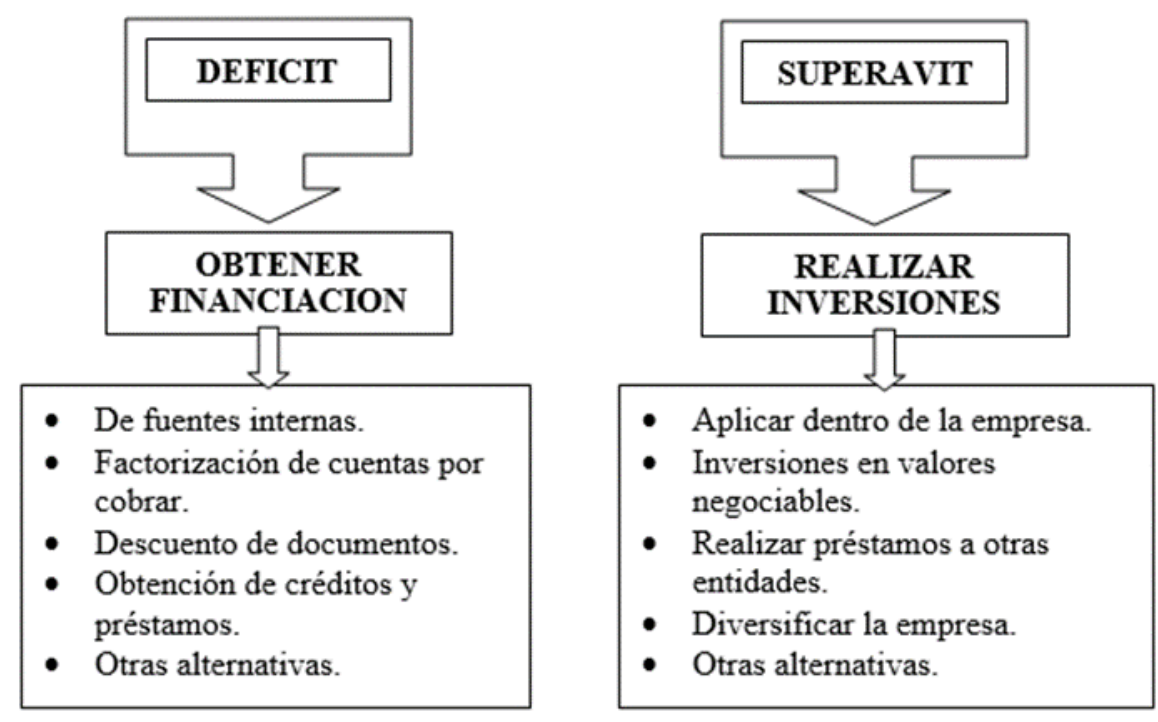

Operaciones que producen Entradas y Salidas de Efectivo

Las operaciones que producen entradas $y$ salidas de efectivo se relacionan fundamentalmente con las operaciones de la empresa, pero existen otras transacciones no menos importantes, que producen flujos de efectivo y que se relacionan con las inversiones y la financiación.

Transacciones que producen entradas:

\begin{tabular}{|c|c|}
\hline $\begin{array}{l}\text { Cobros a clientes por ventas al contado de productos, mercancías y servicios. } \\
\text { Cobro a clientes por ventas a crédito de productos, mercancías y servicios. } \\
\text { Cobro de intereses ganados. } \\
\text { Cobro de dividendos. }\end{array}$ & $\begin{array}{l}\text { ACTIVIDADES } \\
\text { DE OPERACIÓN }\end{array}$ \\
\hline $\begin{array}{l}\text { Producto de la venta de certificados de depósitos y valores negociables } \\
\text { Producto de la venta de activos fijos. } \\
\text { Producto de la venta de inversiones financieras a largo plazo. }\end{array}$ & $\begin{array}{l}\text { ACTIVIDADES } \\
\text { DE INVERSION }\end{array}$ \\
\hline $\begin{array}{l}\text { Producto del recaudo de préstamos. } \\
\text { Producto de préstamos a corto plazo obtenidos. } \\
\text { Producto de préstamos a largo plazo obtenidos. } \\
\text { Efectivo recibido de los propietarios. }\end{array}$ & $\begin{array}{c}\text { ACTIVIDADES } \\
\text { DE FINANCIACION }\end{array}$ \\
\hline
\end{tabular}




\section{Propuesta de flujo efectivo con financiamiento utilizando bono o acciones}

para la empresa de fertilizante

Vol. 2, núm. Esp., (2018)

Pedro Ufredo León Cedeño; Jimmy Javier Jara Nivelo; Grace Adalid Luna Yerovi

Transacciones que producen salidas:

Pagos a proveedores por compras al contado de materiales, mercancías y servicios.

Pagos a proveedores por compras a crédito de productos, mercancias y servicios.

Pagos a empleados.

Pagos de intereses causados.

Pagos de impuestos.

Pagos por compras de certificados de depósitos y valores negociables.

Pagos por compras de activos fijos

Desembolsos en inversiones financieras a largo plazo.

Préstamos hechos.

Pagos de los préstamos a corto plazo (sólo el principal).

Pagos de los préstamos a largo plazos (sólo el principal).

Pagos de dividendos a los propietarios.

Pagos por compras de acciones emitidas por la propia compañía (readquisiciones).

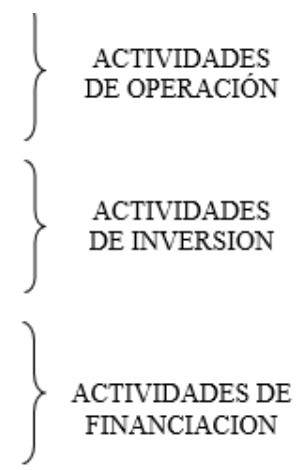

Tabla $N^{\circ} 3$ Estado de Resultados

\begin{tabular}{lcc}
\hline Ingresos y utilidades & Parcial & \multicolumn{1}{l}{ Total } \\
\hline Ventas Netas & $\$ 84.094,84$ & \\
\hline Dividendos Ganados & \\
\hline Intereses Ganados & $\$ 84.094,84$ & \\
\hline Ganancia En Venta De Activos Fijos & \\
\hline Ingresos Y Utilidades Totales & $\$ 35.531,78$ \\
\hline Costos, Gastos Y Pérdidas & $\$ 27.013,49$ & \\
\hline Costo De Mercancías Vendidas & $\$ 5.312,04$ & \\
\hline Gastos Operacionales & $\$ 1.465,14$ & \\
\hline Gastos De Ventas & $\$ 2.865,37$ & \\
\hline Gastos De Depreciación & $\$ 10.181,14$ & \\
\hline Otros Gastos & $\$ 1.000,00$ & \\
\hline Gastos Financieros & & $\$ 725,88$ \\
\hline Gastos De Constitución & & $\$ 159,69$ \\
\hline UTILIDAD DEL EJERCICIO ANTES DE IMPUESTOS & & \\
\hline IMPUESTO & & $\$ 566,19$ \\
\hline $\begin{array}{l}\text { UTILIDAD DEL EJERCICIO ANTES DE PARTICIPACION DE } \\
\text { TRABAJADORES }\end{array}$ & $\$ 84,93$ \\
\hline PARTICIPACION DE TRABAJADORES & $\$ 48,13$ \\
\hline UTILIDAD DEL EJERCICIO ANTES DE RESERVA \\
LEGAL
\end{tabular}


Propuesta de flujo efectivo con financiamiento utilizando bono o acciones para la empresa de fertilizante

Vol. 2, núm. Esp., (2018)

Pedro Ufredo León Cedeño; Jimmy Javier Jara Nivelo; Grace Adalid Luna Yerovi

Gráfico $N^{\circ} 4$ Procedimiento general para la elaboración del Estado de Flujo de Efectivo

\begin{tabular}{|l||l|l|}
\hline ESTADO DE \\
RESULTADOS \\
INGRESOS
\end{tabular}

Parta facilitar el desarrollo y proyecciones del ejemplo, no obstante es válido recalcar que para el estado de flujo de efectivo es necesario realizar un formato de trabajo para el cálculo de las variaciones, es probable que se necesite información para las diversas actividades de la empresa.

Actividades en operación

1. La empresa obtuvo un total de $\$ 84.094,84$ de ingresos por la comercialización de fertilizantes.

2. La empresa no posee otra fuente que le ayude a generar otro ingreso, debido a esto la empresa no otorga créditos en las compras para evitar que las cuentas por cobras sean considerablemente altas y que estas no se conviertan en efectivo.

3. La depreciación de activos obtuvo un total de $\$ 1.465,14$.

4. Los costos y gasto de la empresa suman un valor de $\$ 71.722,68$. 


\section{Propuesta de flujo efectivo con financiamiento utilizando bono o acciones \\ para la empresa de fertilizante}

Vol. 2, núm. Esp., (2018)

Pedro Ufredo León Cedeño; Jimmy Javier Jara Nivelo; Grace Adalid Luna Yerovi

Actividades de inversión

1. Si es necesaria la compra de nuevos bienes como muebles, maquinarias o herramientas, se tiene proyectado realizar la adquisición mediante préstamos bancarios.

Actividades de financiación

1. Durante el año la empresa cancelo un valor de $\$ 10.181,14$ por concepto de préstamo bancarios a corto plazo.

2. Debido a la baja liquidez que la empresa posee al finalizar el año contable, se plantea la idea de la venta de acciones para que la empresa obtenga otra fuente de ingresos para que pueda expandirse y penetre en otros mercados.

\section{Tabla $N^{\circ} 4$ Flujo de efectivo con financiamiento a largo plazo}

\begin{tabular}{|c|c|c|c|c|c|c|c|c|c|c|}
\hline ITEMS & Año 1 & Año 2 & Año 3 & Año 4 & Año 5 & Año 6 & Año 7 & Año 8 & Año 9 & Año 10 \\
\hline SALDO INICIAL & & $\$ 472,00$ & $\$ 1.348,84$ & $\$ 8.407,16$ & $\$ 12.493,28$ & \$15.690,91 & $\$ 16.913,15$ & $\$ 16.602,27$ & \$ 15.398,19 & $\$ 14.691,12$ \\
\hline VENTAS & & & & & & & & & & \\
\hline $\begin{array}{r}\text { CONTADO } \\
\text { CRÉDITO }\end{array}$ & $\$ 84.094,84$ & $\$ 85.776,74$ & $\$ 87.492,27$ & $\$ 87.579,76$ & $\$ 90.278,85$ & $\$ 90.463,49$ & $\$ 90.576,85$ & $\$ 90.913,15$ & $\$ 91.269,45$ & $\$ 91.537,43$ \\
\hline OTROS INGRESOS & & $\$ 1.500,00$ & $\$ 2.000,00$ & $\$ 1.500,00$ & $\$ 1.000,00$ & $\$ 2.500,00$ & $\$ 1.500,00$ & $\$ 2.000,00$ & $\$ 2.500,00$ & $\$ 2.700,00$ \\
\hline TOTAL DE INGRESOS & $\$ 84.094,84$ & $\$ 87.276,74$ & $\$ 89.492,27$ & $\$ 89.079,76$ & $\$ 91.278,85$ & $\$ 92.963,49$ & $\$ 92.076,85$ & $\$ 92.913,15$ & $\$ 93.769,45$ & $\$ 94.237,43$ \\
\hline COMPRAS & & & & & & & & & & \\
\hline $\begin{array}{r}\text { CONTADO } \\
\text { CRÉDITO }\end{array}$ & $\$ 35.531,78$ & $\$ 36.597,73$ & $\$ 37.329,69$ & $\$ 39.196,17$ & $\$ 41.155,98$ & $\$ 43.213,78$ & $\$ 44.078,06$ & $\$ 44.959,62$ & $\$ 45.858,81$ & $\$ 46.775,99$ \\
\hline GASTOS & & & & & & & & & & \\
\hline GASTOS OPERACIONALES & $\$ 27.013,49$ & $\$ 28.094,03$ & $\$ 29.217,79$ & $\$ 28.633,44$ & $\$ 28.378,86$ & $\$ 28.520,75$ & $\$ 28.663,36$ & $\$ 28.806,67$ & $\$ 28.950,71$ & $\$ 29.095,46$ \\
\hline GASTOS DE VENTAS & $\$ 5.312,04$ & $\$ 5.338,60$ & $\$ 5.386,65$ & $\$ 4.309,32$ & $\$ 4.093,85$ & $\$ 3.889,16$ & $\$ 3.694,70$ & $\$ 3.937,48$ & $\$ 3.740,61$ & $\$ 3.553,58$ \\
\hline GASTOS DE DEPRECIACIÓN & $\$ 1.465,14$ & $\$ 1.344,17$ & $\$ 1.492,02$ & $\$ 1.193,62$ & $\$ 1.133,94$ & $\$ 1.077,24$ & $\$ 1.023,38$ & $\$ 2.188,26$ & $\$ 2.078,85$ & $\$ 1.974,90$ \\
\hline OTROS GASTOS & $\$ 2.865,37$ & $\$ 2.894,03$ & $\$ 2.914,29$ & $\$ 2.943,43$ & $\$ 2.972,86$ & $\$ 3.002,59$ & $\$ 3.032,62$ & $\$ 3.062,94$ & $\$ 3.093,57$ & $\$ 3.124,51$ \\
\hline GASTOS FINANCIEROS & $\$ 10.181,14$ & $\$ 10.181,14$ & & & & & & & & \\
\hline GASTOS DE CONSTITUCIÓN & $\$ 1.000,00$ & $\$ 1.200,00$ & $\$ 1.260,00$ & $\$ 1.295,00$ & $\$ 1.310,00$ & $\$ 1.315,00$ & $\$ 1.370,00$ & $\$ 1.400,00$ & $\$ 1.440,00$ & $\$ 1.450,00$ \\
\hline TOTAL DE GASTOS & $\$ 47.837,19$ & $\$ 49.051,97$ & $\$ 40.270,75$ & $\$ 38.374,80$ & $\$ 37.889,51$ & $\$ 37.804,75$ & $\$ 37.784,06$ & \$ $39.395,36$ & $\$ 39.303,73$ & $\$ 39.198,45$ \\
\hline SALDO FINAL & $\$ 725,87$ & $\$ 2.099,03$ & $\$ 13.240,67$ & $\$ 19.915,95$ & $\$ 24.726,63$ & $\$ 27.635,87$ & $\$ 27.127,89$ & $\$ 25.160,44$ & $\$ 24.005,10$ & $-\$ 22.954$ \\
\hline
\end{tabular}




\section{Propuesta de flujo efectivo con financiamiento utilizando bono o acciones para la empresa de fertilizante}

Vol. 2, núm. Esp., (2018)

Pedro Ufredo León Cedeño; Jimmy Javier Jara Nivelo; Grace Adalid Luna Yerovi

\section{Validación de la propuesta}

Al leer el estado de pérdidas y ganancias, el gerente debe entender las características de flujo de efectivo de ingresos y gastos. Deben tener en cuenta que el contador registra los ingresos por ventas cuando se realizan ventas independientemente cuando se recibe dinero en efectivo de los clientes.

Los gastos de registros de contador para que coincida con los gastos con los ingresos por ventas y los gastos para poner en el período al que pertenecen - aparte de cuándo se pagan en efectivo para los gastos. El gerente no debe suponer que los ingresos por ventas es igual a la entrada de efectivo, y que los gastos de la misma salida de caja.

Las acciones que se venden dentro del mercado de valores se realizara siempre y cuando se observe que los niveles de liquidez de la empresa mejoren, de esta manera se puede generar nuevas relaciones comerciales las cuales ayudaran a obtener nuevos ingresos que beneficien a la empresa.

\section{Conclusiones.}

Se pudo constatar los problemas financieros de la empresa, el implementar una herramienta que ayude en la gestión de efectivo, de esta manera la empresa obtenga una mejor liquidez al finalizar el periodo contable.

Se determina que la empresa lleva un inadecuado proceso para el control de los gastos y/o ingresos que se realizan las actividades comerciales, debido a que no realiza constataciones de manera física, en reiteradas ocasiones los valores que se registran en los balances son asumidos 


\section{Propuesta de flujo efectivo con financiamiento utilizando bono o acciones para la empresa de fertilizante}

Vol. 2, núm. Esp., (2018)

Pedro Ufredo León Cedeño; Jimmy Javier Jara Nivelo; Grace Adalid Luna Yerovi

por parte del personal competente, lo que ocasiona un desconocimiento del manejo de capital que realiza la empresa.

Debido al mal manejo de registros que realiza el personal implicado en esta actividad, se puede asumir que no está totalmente capacitado para el cargo o desconoce todo el procedimiento contable que ayude a reflejar la situación económica, financiera y tributaria de la empresa.

Las herramientas utilizadas como técnicas de investigación, ayudaron a determinar e identificar los problemas que afronta la empresa, se obtuvo como resultado la estructura actual y el proceso contable que maneja el personal competente de la empresa, estas técnicas dieron las pautas para priorizar el problema y determinar una propuesta que ayude a mejorar la situación que afrontan el cual es debido principalmente a la falta de ingresos.

\section{Bibliografía.}

Durán, Y. (2011). Administración del Capital de Trabajo: una herramienta financiera para la gerencia de las PyME tradicionales venezolanas. Visión Gerencial, 1(ene), 37-56.

Finnerty, J. (1998). Financiamiento de proyectos. México: Pearson, Prentice Hall.

Garrido, C., \& Granados, L. (2004). Innovación, financiamiento y organización financiera nacional. Problemas del Desarrollo. Revista Latinoamericana de Economía, 35(139), $163-184$.

Páez, T. (2007). eoría y práctica de la ética y la responsabilidad social de la PyME venezolana. Revista FACES, 17(5), 165-183.

Vargas Soto, R. (2007). ESTADO DE FLUJO DE EFECTIVO. InterSedes: Revista de las Sedes Regionales, 7(14), 111-136. 